\title{
First molecular subtyping and phylogeny of Blastocystis sp. isolated from domestic and synanthropic animals (dogs, cats and brown rats) in southern Iran
}

\author{
Iraj Mohammadpour ${ }^{*}$ (D, Farzaneh Bozorg-Ghalati ${ }^{2}$, Alessia Libera Gazzonis ${ }^{3 *}$, Maria Teresa Manfredi ${ }^{3}$,
} Mohammad Hossein Motazedian ${ }^{1}$ and Niloofar Mohammadpour ${ }^{4}$

\begin{abstract}
Background: Blastocystis sp. is a common intestinal protist that infects humans and many animals globally. Thus far, 22 subtypes (STs) have been identified in mammalian and avian hosts. Since various STs are common to humans and animals, it was suggested that some human infections might arise from zoonotic transmission. Therefore, the aim of this study was to assess the presence of Blastocystis sp. in domestic (dogs and cats) and synanthropic animals (rats) of Fars Province, Iran, and to genetically characterize the samples.

Methods: A total of 400 fresh faecal samples from 154 dogs, 119 cats, and 127 rats were inspected by direct microscopy, Wheatley's trichrome staining, in vitro culture, and 185 rRNA gene nested-PCR. Finally, sequencing and phylogenetic analyses were performed.

Results: Out of 400 samples, 47 (11.8\%) and 61 (15.3\%) samples were detected as positive by direct wet mount and culture, respectively. Molecular analysis detected a larger number of positive samples ( $n=70,17.5 \%$ ): nested-PCR showed that 29 (18.8\%) dogs, 21 (17.7\%) cats, and 20 (15.8\%) rats were infected by Blastocystis sp. Sequence analysis of positive samples indicated the presence of zoonotic STs in all investigated host species. Specifically, ST2 (allele 9), ST3 (allele 34), ST4 (allele 94), ST7 (allele 99), ST8 (allele 21), and ST10 (allele 152) were detected in dogs; ST1 (allele 2), ST3 (allele 34), ST4 (allele 94), ST10 (allele 152), and ST14 (allele 159) were detected in cats; and ST1 (allele 2), ST3 (allele 34), and ST4 (allele 92) were detected in rats.
\end{abstract}

Conclusions: Our data suggest that domestic dogs and cats can serve as possible reservoirs for in-contact humans, especially those who handle shelter-resident and client-owned animals. Moreover, rats as synanthropic animals can function as a potential source of human infections. Conversely, humans can act as a source of infections to animals. These results should be reinforced in future molecular epidemiological studies.

Keywords: Blastocystis, Subtyping, Phylogenetic analysis, Canis lupus familiaris, Felis catus domesticus, Rattus norvegicus, Iran

\footnotetext{
*Correspondence: mohamad_i@sums.ac.ir; alessia.gazzonis@unimi.it

${ }^{1}$ Department of Medical Parasitology and Mycology, School of Medicine,

Shiraz University of Medical Sciences, Shiraz, Iran

${ }^{3}$ Department of Veterinary Medicine, Università degli Studi di Milano, Milan, Italy

Full list of author information is available at the end of the article
}

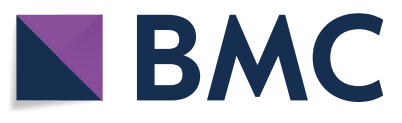

(c) The Author(s) 2020. This article is licensed under a Creative Commons Attribution 4.0 International License, which permits use, sharing, adaptation, distribution and reproduction in any medium or format, as long as you give appropriate credit to the original author(s) and the source, provide a link to the Creative Commons licence, and indicate if changes were made. The images or other third party material in this article are included in the article's Creative Commons licence, unless indicated otherwise in a credit line to the material. If material is not included in the article's Creative Commons licence and your intended use is not permitted by statutory regulation or exceeds the permitted use, you will need to obtain permission directly from the copyright holder. To view a copy of this licence, visit http://creativeco mmons.org/licenses/by/4.0/. The Creative Commons Public Domain Dedication waiver (http://creativecommons.org/publicdomain/ zero/1.0/) applies to the data made available in this article, unless otherwise stated in a credit line to the data. 


\section{Background}

Blastocystis sp. is an anaerobic eukaryotic protist belonging to the phylum Stramenopiles. It is isolated from the lumen of humans and animals and has four different morphological forms (central-body, granular, amoeboid and cystic) [1]. Binary fission, budding, and plasmotomy are the forms of reproduction observed in Blastocystis sp. [2]. Blastocystis sp. has a global distribution, with a recently reported prevalence of up to $100 \%$ in humans [3]. It is believed that Blastocystis sp. is transmitted via the faecal-oral route, water, food, and direct person-toperson contact $[1,4]$. There is supporting evidence that zoonotic transmission of Blastocystis sp. from animals to humans living in a community-based environment may occur via close contact with animal enclosures [5-7].

In recent years, several studies have reported that Blastocystis sp. is a causative agent of diarrhoea, abdominal pain, flatulence, irritable bowel syndrome, inflammatory bowel disease, and urticaria [8, 9]. However, its pathogenicity is still a topic of some debate, as asymptomatic carriage is common $[10,11]$. Considerable evidence suggests that Blastocystis sp. is capable of producing cysteine proteinases that interpose interleukin-8 release from enterocytes, impelling enterocytes apoptosis and augmenting gut permeability while also potentially evading recognition by Toll-like receptors $[12,13]$.

Blastocystis sp. has been shown to exhibit extensive genetic diversity [14, 15]. Hitherto, 22 different subtypes (STs) of Blastocystis sp., consisting of ST1 to ST17, ST21, and ST23 to ST26 have been described in humans and a variety of animals based on polymorphisms in $18 \mathrm{~S}$ rRNA gene sequences [16]. Nevertheless, the prevalence of the different subtypes varies among countries and among regions within the same country. ST1 to ST9 and ST12 have been isolated from humans, with ST3 being the most frequent subtype, and all except ST9 have also been reported in animals $[17,18]$. ST5 is prevalently isolated from livestock, ST6 and ST7 from birds, and ST8 from non-human primates (NHPs) [19-22]. The proof that ST5 to ST8 have only been fortuitously discovered in humans has been interpreted as suggestive of zoonotic transmission [19-22]. ST10 to ST17, ST21, and ST23 to ST26 have been recorded only in non-human hosts thus far [14-16].

A substantial number of animal species have been examined for the identification of Blastocystis sp. [5, 14, 20-23]. However, more studies are required to characterize the zoonotic transmission of this parasite and to identify whether other subtypes of Blastocystis sp. exist. The analysis of DNA extracted directly from stool samples is considered to be highly sensitive, providing the means for genotyping and subtyping [24, 25]. Diagnostic real-time
PCR has been recently introduced; however, so far it has not been used for screening [26].

Blastocystis sp. has been reported to have prevalence rates of $2-47 \%$ in livestock and $8-67 \%$ in captive animals from zoological gardens [14, 20-22, 27]. Presently, an expanding interest in Blastocystis sp. research fortifies the necessity for investigations of this parasite in areas where it has not yet been assessed. In Iran, molecular studies were performed in relation to human infection [28]. Concerning animals, Blastocystis sp. was previously genotyped from hooded crows and pigeons [29], cattle [30] and wild boars [31], while there are no molecular studies regarding the occurrence of Blastocystis sp. in animals living in close contact with humans, such as pets and synanthropic animals. Therefore, the aim of this study was to assess the presence of Blastocystis sp. in dogs, cats and rats of Fars Province, southern Iran, and to genetically characterize the samples by $18 S$ rRNA gene sequencing. To the best of our knowledge, this is the first and largest epidemiological study executed on these animals in Iran.

\section{Methods \\ Sample collection}

For the present cross-sectional study, 400 fresh faecal samples from 154 dogs (Canis lupus familiaris), 119 cats (Felis catus domesticus), and 127 brown rats (Rattus norvegicus) were collected from December 2016 to October 2018. The study was performed on dogs and cats regardless of their race, age or sex.

Dogs' fresh faecal samples were collected individually per-rectum under an anaesthesia regime during a trap, neuter, and release (TNR) sterilization programme to restrain stray dog populations controlled by the Fars Veterinary Administration.

After consulting with the vector control unit of the Fars Veterinary Administration, searching and identifying the active colonies of rodents in different places of Fars Province were carried out and wild rodents were trapped. Cats and brown rats were lured in the same spatiotemporal location using Sherman baited cage-traps with tinned fish and roasted walnuts, respectively. Wire cage-traps were placed at rodents' burrows entrances in gardens and grasslands around the residential houses in different cities and counties of Fars Province. Traps were set in the evening and were checked the next day in the morning. Faeces of captive animals was collected from their cages to minimize contamination using disposable spoons, stored in tagged polystyrene flasks, and transported as early as possible to the head laboratory. Gross macroscopic examination of faecal samples was done to check for consistency. The consistencies of the collected faecal 
specimens were formed and soft. Every time a mean of 10 traps was used for screening and processing.

\section{Microscopic examination}

Samples were processed within $2-4$ h after collection. Faecal smears were prepared and first examined by direct microscopy using both a saline and iodine wet mount preparation. In addition, faecal smears were stained with Wheatley's trichrome stain.

\section{Faecal culture}

In vitro culture was concurrently carried out for all stool samples using Jones' medium supplemented with $10 \%$ heat-inactivated horse serum (Gibco, Frankfurt, Germany), $10 \%$ Pen-Strep $(1000 \mathrm{IU} / \mathrm{ml}$ and $500 \mu \mathrm{g} / \mathrm{ml})$ and powdered rice-starch. Nearly $200 \mathrm{mg}$ stool specimens were inoculated into $5 \mathrm{ml}$ screw-cap tubes containing $3 \mathrm{ml}$ of Jones' medium using a sterile disposable applicator stick. The culture tubes were incubated at $37^{\circ} \mathrm{C}$ for $72 \mathrm{~h}$ [32]. The presence of any of the 4 morphologies of Blastocystis sp. was observed daily by placing one drop of culture product onto SAF-coated coverslips and staining with Wheatley's trichrome stain. Positive samples were subcultured on LYSGM medium supplemented with 10\% heat-inactivated horse serum (Gibco) for mass cultivation [32].

\section{DNA extraction}

To lessen impediments from raw fibres and impurities, the faecal specimens were sieved and washed 3 times with distilled water by centrifugation at $1500 \times g$ for 10 min. Total genomic DNA of Blastocystis sp. was extracted using a QIAamp ${ }^{\circledR}$ Fast DNA Stool Mini Kit (Qiagen, Hilden, Germany) according to the manufacturer's instructions. DNA quality was verified by NanoDrop ${ }^{\circledR}$ (Thermo Fisher Scientific, Carlsbad, CA, USA) measurements. The final DNA was eluted in $100 \mu \mathrm{l}$ of $\mathrm{AE}$ buffer to increase its concentration and stored at $-20^{\circ} \mathrm{C}$ until use.

\section{Nested-PCR amplification}

Amplification of a 1100-bp fragment of the Blastocystis sp. 18S rRNA gene was carried out using a two-step nested-PCR. This method was chosen since it provides a large fragment for precise sequencing and phylogenetic analysis, and as it is a pan-Blastocystis sp. technique, it permits the identification of known and unknown subtypes of Blastocystis sp. [33].

The primers RD3 (5'-GGG ATC CTG ATC CTT CCG CAG GTT CAC CTA C-3'); RD5 (5'-GGA AGC TTA TCT GGT TGA TCC TGC CAG TA-3'); BIF (5'-GGA GGT AGT GAC AAT AAA TC-3'); and BIR (5'-CGT TCA TGA TGA ACA ATT AC-3') were used for the first and second rounds of the nested-PCR. The first PCR reactions were performed in $25 \mu \mathrm{l}$ reaction mixtures containing $1 \times$ PCR buffer $(10 \mathrm{mM}$ Tris- $\mathrm{HCl}, \mathrm{pH} 9,50 \mathrm{mM}$ $\mathrm{KCl}$ ), $1.5 \mathrm{mM} \mathrm{MgCl}, 0.2 \mathrm{mM}$ of each dNTPs (Roche, Alameda, CA, USA), 1.25 U Platinum $^{\circledR}$ Taq High-Fidelity DNA polymerase (Invitrogen, Groningen, the Netherlands), $40 \mu \mathrm{g} / \mathrm{ml}$ of BSA, $10 \mathrm{pM}$ of each forward and reverse primers, and $2 \mu \mathrm{l}$ genomic DNA.

The PCR conditions consisted of pre-denaturation at $95{ }^{\circ} \mathrm{C}$ for $5 \mathrm{~min}, 40$ cycles of denaturation at $95{ }^{\circ} \mathrm{C}$ for $30 \mathrm{~s}$, annealing at $65{ }^{\circ} \mathrm{C}$ for $45 \mathrm{~s}$, extension at $72{ }^{\circ} \mathrm{C}$ for $1 \mathrm{~min}$ and a final extension at $72{ }^{\circ} \mathrm{C}$ for $10 \mathrm{~min}$ using an Eppendorf Mastercycler Gradient PCR machine (Eppendorf, Hamburg, Germany). The second PCR cycle was carried out with similar reaction systems except using the PCR products of the first cycle as the template, and $54{ }^{\circ} \mathrm{C}$ as the annealing temperature. Electrophoresis was performed by adding $2 \mu \mathrm{l}$ of the PCR products and a 100-bp molecular marker (GenScript, Tokyo, Japan) loaded on $1.5 \%$ agarose gels (AddGene, Watertown, MA, USA) in TBE buffer and staining with SYBR ${ }^{\circledR}$ Safe DNA Gel Stain (Thermo Fisher Scientific) for $1 \mathrm{~h}$ at $90 \mathrm{~V}$. Bands were observed by UV light and photographed (Uvitec, Cambridge, UK). Positive and negative controls were included in every PCR run.

\section{Sequencing and phylogenetic analysis}

Bands of the expected size were excised from the agarose gel and purified using a QIAquick ${ }^{\circledR}$ Gel Extraction Kit (Qiagen) according to the manufacturer's instructions. Purified products were sequenced in both directions using nest-2 primers at Roche Molecular Diagnostics (Roche, Mannheim, Germany) by capillary electrophoresis using a Big Dye ${ }^{\mathrm{TM}}$ Terminator Cycle Sequencing Kit (Applied Biosystems, Foster City, CA, USA) in an ABI PRISM $^{\circledR} 3730$ automated sequencer.

Raw sequencing data in both the forward and reverse directions were checked using Chromas 2.6.6 (Technelysium, Brisbane, Australia). Special attention was paid to the double peaks (indicative of mixed infections by different subtypes or alleles) and the accuracy of the nucleotides was guaranteed. Multiple sequence alignment was performed using the MUSCLE algorithm of MEGA-X [34]. The consensus sequences were then compared with homologous sequences available in the GenBank database using the nBLAST program. The sequences were assembled and edited with BioEdit (v.7.2.6; https://www. bioedit.com). Subtypes were determined by exact match or an identity $\geq 98 \%$ against all known Blastocystis sp. subtypes, with a query coverage of $\geq 98 \%$.

All established sequences were submitted to the multilocus sequence typing (MLST) database (https://pubml 
st.org/blastocystis/) for subtype confirmation and relevant allele identification.

Two molecular phylogenetic trees (one for dogs and cats, and another for rats) were constructed with the Neighbour-Joining (NJ) method, and genetic distances were calculated with the Maximum Composite Likelihood model in MEGA-X [34]. Bootstrap analysis (with 1000 replicates) was carried out to define the robustness of the findings. Proteromonas lacertae and Blastocystis lapemi were used as outgroups for the two analyses.

\section{Statistical analysis}

The agreement between microscopy, culture, and PCR results was measured using the Cohen's kappa coefficient (к) (GraphPad Prism ${ }^{\circledR}$, Melbourne, Australia) and results were interpreted as follows: no agreement $(\kappa \leq 0)$; slight agreement $(0.01<\kappa<0.2)$; fair agreement $(0.21<\kappa<0.4)$; moderate agreement $(0.41<\kappa<0.6)$; substantial agreement $(0.61<\kappa<0.8)$; and almost perfect agreement $(0.81<\mathrm{K} \leq 1)$.

Fisher's exact test was used to compare the frequency of Blastocystis sp. carriage between dogs, cats, and rats (based on molecular results). The significance level was established at $P<0.05$.

\section{Results}

\section{Infection rates, subtyping and allele analysis}

Overall, 400 faecal samples were collected in the current study from 154 dogs, 119 cats, and 127 rats and investigated for Blastocystis sp. Out of 400 faecal samples, 47 (11.8\%) samples were shown to be positive by direct wet mount and Wheatley's trichrome staining examination. The most common finding was the central-body form of the protozoan (Additional file 1: Figure S1). Among 400 stool samples, 61 (15.3\%) were found to be positive by in vitro cultivation in Jones' medium. The most common forms of Blastocystis sp. in Jones' medium were the central-body and granular forms. K-values of $0.461,0.706$ and 0.864 were appraised between microscopy, culture, and PCR (Table 1).

Nested-PCR was carried out for amplification of a 1100-bp band (Additional file 2: Figure S2). Overall, 29 faecal samples from dogs (18.8\%), 21 faecal samples from cats $(17.7 \%)$, and 20 faecal samples from rats (15.8\%) were detected as positive by nested-PCR (Table 2). No statistically significant differences in prevalence values were recorded among the three species studied $(P>0.05)$. All positive samples were sequenced successfully. The partial sequences of the $18 S$ rRNA gene of Blastocystis sp. obtained in this study were deposited in the GenBank database under accession numbers MN264509-MN264522.
Among the 70 Blastocystis-positive samples, 8 subtypes were detected: 6 zoonotic STs (ST1-ST4, ST7, and ST8), ST10, and ST14. Dogs were the hosts to the widest range of STs, followed by cats and rats. Remarkably, 6 subtypes were found in the dogs, including ST2 (allele 9), ST3 (allele 34), ST4 (allele 94), ST7 (allele 99), ST8 (allele 21), and ST10 (allele 152). Meanwhile, 5 subtypes were detected in the cats, comprising ST1 (allele 2), ST3 (allele 34), ST4 (allele 94), ST10 (allele 152), and ST14 (allele 159). In addition, 3 subtypes were found in the rats, including ST4 (allele 92), ST3 (allele 34), and ST1 (allele 2) (Table 2). No mixed infections involving different STs of the protist were identified. No intra-subtype genetic heterogeneity was observed within detected subtypes.

\section{Phylogenetic analysis}

A total of 14 representative sequences were obtained from 70 Blastocystis sp. isolates in the present study (Table 2). The sequences obtained in this survey shared high identity with the Blastocystis sp. sequences registered in GenBank.

When the phylogenetic analysis was executed, in contrast to the 17 reference subtype sequences in GenBank, we procured a precise discrimination of Blastocystis sp. subtypes compatible with that established by BLAST queries. Because our study confirmed that ST4 was the most dominant subtype in southern Iranian rodent population, so two phylogenetic trees were constructed to compare this subtype with other rat-derived ST4. All sequences obtained from rats, cats, and dogs were closely related to animal- or human-derived sequences in GenBank and clustered together (Figs. 1 and 2).

Inter-subtype genetic polymorphism was discerned between detected subtypes and reference subtypes registered in GenBank. According to homology analysis, the two ST1 sequences derived from rats and cats (MN264511 and MN264518, respectively) were the same and were $99.36 \%$ and $98.91 \%$ similar to those from pigs in France (AY135404) and from humans in Japan (AB023499). The only ST2 sequence obtained from dogs (MN264512) shared $99.36 \%$ and $98.27 \%$ identity with that from pigs in the Philippines (EU445487) and from monkeys in Japan (AB107969). Among the three ST3 sequences, one rat-derived and one cat-derived Blastocystis sp. isolate produced the same sequences (MN264510 and MN264519, respectively), with these sequences being $99.45 \%, 99.36 \%$, and $99.36 \%$ similar to those in cattle in Japan (AB107965), humans in the Philippines (EU445496), and pigs in Germany (MK801366), respectively. The remaining dog-derived ST3 sequence (MN264513) was 99.27\% similar to that from dogs in Malaysia (KX234611). 
Among the three ST4 isolates, three different sequences were obtained. The rat-derived ST4 sequence (MN264509) was $99.36 \%$ and $99.27 \%$ similar to that from rats in France (AY135408) and from rats in Japan (AB071000 and MH127499), respectively. The catderived ST4 sequence (MN264520) shared 99.36\% identity with that from humans in the USA (JN682513) and from rats in Indonesia (MH127488). The remaining dogderived ST4 sequence (MN264514) was 99.36\% similar to that from dogs in Malaysia (KX234618). The only dogderived ST7 sequence (MN264516) was 99.36\% similar to that from chickens in France (AY135410). In addition, the only dog-derived ST8 sequence (MN264515) was 99.36\% similar to that from dogs in Malaysia (KX234612).

Among the two ST10 isolates, two different sequences were obtained. The dog-derived ST10 sequence (MN264517) shared $99.36 \%$ identity with that from dogs in Malaysia (KX234616). The cat-derived ST10 sequence (MN264521) was $99.36 \%$ similar to that from camels in Libya (KC148207) and from goats in Malaysia (KX234638). The only cat-derived ST14 sequence (MN264522) shared 99.27\% identity with that from cattle in England (KC148205).

Table 1 The estimated k-value between microscopy, culture, and PCR for identification of Blastocystis sp

\begin{tabular}{lll}
\hline Method & k-value & Degree of agreement \\
\hline Microscopy & 0.461 & Moderate \\
Culture & 0.706 & Substantial \\
PCR & 0.864 & Perfect \\
\hline
\end{tabular}

\section{Discussion}

Blastocystis sp. is an enteric protist with a universal distribution. It is assumed that multiple zoonotic isolates of Blastocystis sp. exist with regular animal-to-human and human-to-animal transmission and as a large potential reservoir in animals for infections in humans [4, 35-37]. Recent genomic data revealed putative virulence factors and illustrated deleterious clinical outcomes of Blastocystis sp. on the intestinal barrier, leading to conceivable models of pathogenesis [10-13, 38].

Blastocystis sp. has been secluded from a large spectrum of animals, such as birds, pigs, cattle, sheep, goats, wild carnivores, NHPs, and less frequently rodents, amphibians, reptiles, and insects [21-23, 39-41]. Blastocystis sp. has cryptic host specificity and is heeded potential zoonotic protozoa since infections in humans have been connected with contact with various mammals and birds [21, 42, 43]. Furthermore, successful experimental infections of rats after oral administration of ST1, ST3, and ST4 purified cysts isolated from human stool samples confirmed the transmission of this protist between human and animal hosts $[44,45]$. A higher risk of Blastocystis sp. infection was found in people with close animal contact, supporting the hypothesis of transmission from animals to humans $[5,6,46,47]$.

In a territory where Blastocystis sp. is largely present in humans, there is a necessity to determine the load of this infection in animals and to identify the reservoirs or sources of infection in the community. In Iran, several related studies have been accomplished on the human population [28], while few surveys have been performed for domestic and wild animals [29-31]. To the best of our knowledge, our study represents the first inspection of

Table 2 Percentage of infection and subtype/allele distributions of Blastocystis sp. detected in dogs, cats, and rats in Fars Province, Iran

\begin{tabular}{|c|c|c|c|}
\hline Host species & Positive/examined (\%) & Subtype/alleles (n) & GenBank ID \\
\hline \multirow[t]{3}{*}{ Brown rat (Rattus norvegicus) } & 20/127 (15.8) & ST1/ allele $2(n=4)$ & MN264511 \\
\hline & & ST3/allele $34(n=4)$ & MN264510 \\
\hline & & ST4/allele $92(n=12)$ & MN264509 \\
\hline \multirow[t]{5}{*}{ Cat (Felis catus domesticus) } & $21 / 119(17.7)$ & ST1/allele $2(n=5)$ & MN264518 \\
\hline & & ST3/allele $34(n=7)$ & MN264519 \\
\hline & & ST4/allele $94(n=4)$ & MN264520 \\
\hline & & ST10/allele $152(n=3)$ & MN264521 \\
\hline & & ST14/allele $159(n=2)$ & MN264522 \\
\hline \multirow[t]{6}{*}{ Dog (Canis lupus familiaris) } & 29/154 (18.8) & ST2/allele $9(n=8)$ & MN264512 \\
\hline & & ST3/allele $34(n=11)$ & MN264513 \\
\hline & & ST4/allele $94(n=3)$ & MN264514 \\
\hline & & ST7/allele $99(n=3)$ & MN264516 \\
\hline & & ST8/allele $21(n=2)$ & MN264515 \\
\hline & & ST10/allele $152(n=2)$ & MN264517 \\
\hline
\end{tabular}


and report on the prevalence of Blastocystis sp. subtypes isolated from domestic and synanthropic animals (dogs, cats and brown rats) in Fars Province, Iran. Three diagnostic investigations were conducted in parallel on the 400 faecal samples included in the present study: direct microscopy; in vitro culture; and nested-PCR. Studies have shown that PCR is a valid technique with high sensitivity, specificity, reliability and reproducibility [24]. In the present study, nested-PCR allowed us to identify the largest number of positive samples. Previous studies carried out in animals in Iran showed higher infection rates in hooded crows and pigeons $(42.9 \%$ and $44.4 \%$, respectively) [29] and in wild boars (25.0\%) [31], although a lower infection rate was reported in cattle (9.6\%) [30].

Globally, epidemiological studies conducted on dogs and cats to investigate their potential role as natural

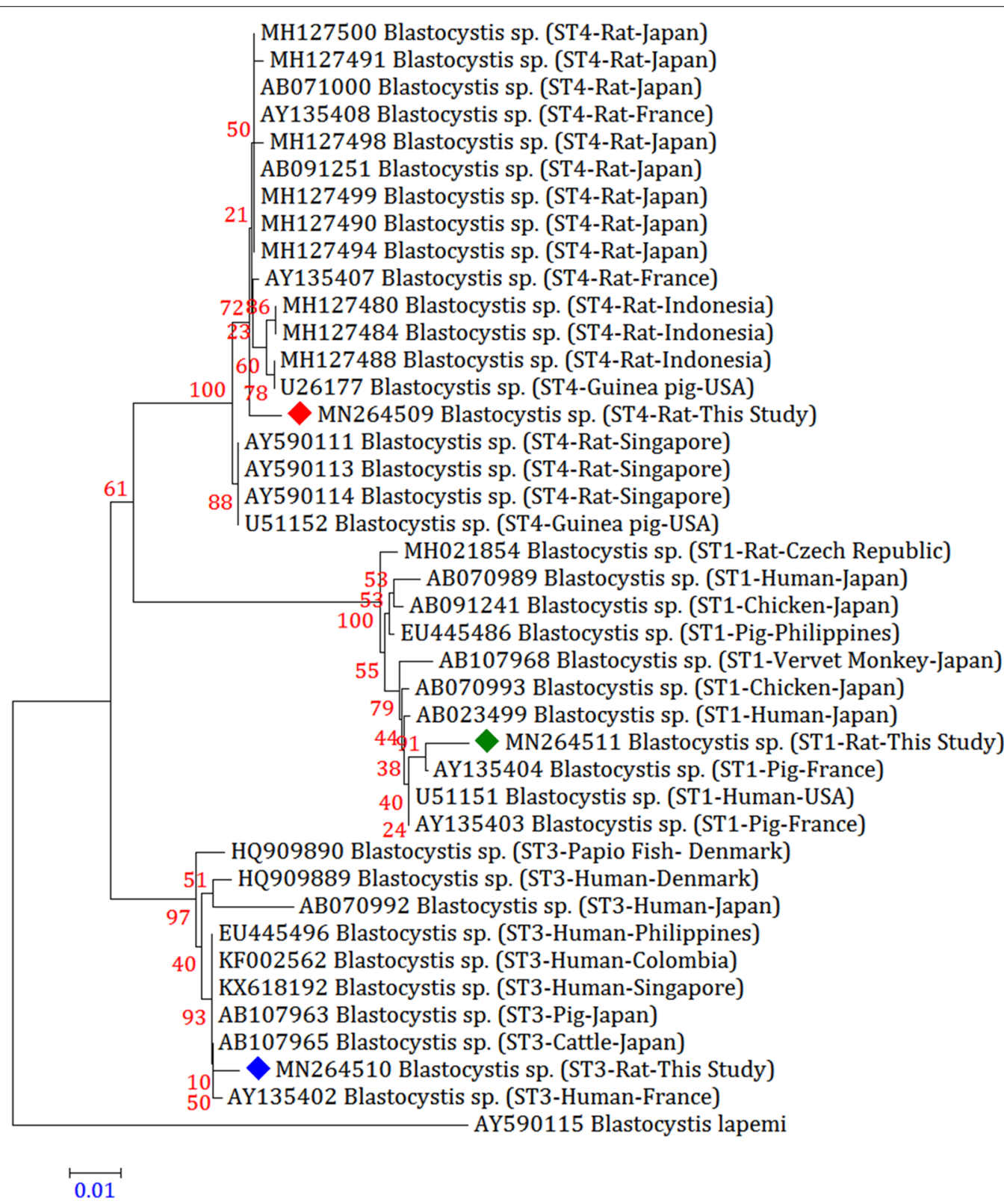

Fig. 1 Molecular phylogenetic relationships between various Blastocystis sp. samples isolated from rats as inferred by the Neighbour-Joining tree based on the $18 \mathrm{~S}$ rRNA gene. The numbers on branches are percentage bootstrap values of 1000 replicates. The evolutionary distances between sequences were computed using the Maximum Composite Likelihood method. The scale-bar indicates an evolutionary distance of 0.01 nucleotides per position in the sequence. The reference sequence accession numbers are shown. Evolutionary analyses were conducted in MEGA-X. Blastocystis lapemi was used as the outgroup 
(See figure on next page.)

Fig. 2 Molecular phylogenetic relationships between various Blastocystis sp. samples isolated from cats and dogs as inferred by the Neighbour-Joining tree based on the $18 \mathrm{~S}$ rRNA gene. The numbers on branches are percentage bootstrap values of 1000 replicates. The evolutionary distances between sequences were computed using the Maximum Composite Likelihood method. The scale-bar indicates an evolutionary distance of 0.02 nucleotides per position in the sequence. The reference sequence accession numbers are shown. Evolutionary analyses were conducted in MEGA-X. Proteromonas lacertae was used as the outgroup

reservoirs of human Blastocystis sp. infection revealed disparate prevalence values. Surveys targeting sheltered and household canine and feline populations did not demonstrate the presence of Blastocystis sp. in Japan and Spain $[48,49]$, while infection rates in the range of 9.7-37\% have been documented in shelter dogs in the USA [7], in stray dogs in India [33], in dogs housed in Italian rescue shelters [50], and in symptomatic dogs and cats attending a veterinary clinic in Chile [51].

Concerning brown rats, the recorded prevalence of $15.8 \%$ was in line with that reported from previous surveys on rodents, ranging from $8.4 \%$ to $45.9 \%[52,53]$. The large number of positive brown rats, synanthropic animals that share spaces with humans and contaminate the environment with faeces and urine, strengthens their role as potential reservoir hosts for Blastocystis sp.

Furthermore, this study is the first to assess the subtype diversity of Blastocystis sp. based on the $18 S$ rRNA gene in dogs, cats and rats in Fars Province, Iran. Currently, human Blastocystis sp. isolates are classified as ST1-ST9 and ST12, and all except ST9 have also been identified in other animals [17, 18, 25, 39]. In particular, ST1 to ST4 are among the most shared subtypes in humans and have been associated with human disease, having low host specificity and probable zoonotic connotation $[54,55]$.

In our study, 8 subtypes were identified: ST1-ST4; ST7; ST8; ST10; and ST14. It is noteworthy that the three investigated species harboured not only the same STs, but also the same alleles of each subtype, indicating a possible common environmental source of infection. ST1, detected in the present study in cats and brown rats, was previously reported in captive wild animals, NHPs, sheep, goats, pigs, water voles, marsupials and birds in various locations [21-23, 27, 56-61]. ST2, recorded in eight dogs, is considered the dominant subtype in NHPs [61]. The zoonotic potential of NHP isolates was previously investigated, in which ST2 isolates with identical sequences were found in rhesus monkeys and children living in the same area of Nepal [46]. Moreover, ST1 and ST2 have been found in zookeepers and five primate species in Australia [5]. ST3 is the Blastocystis sp. subtype with the highest prevalence in humans worldwide and is probably a human species-specific subtype [35, 54, 62]. The finding of ST3 in the three investigated species supported the potential role of domestic and synanthropic animals in human Blastocystis sp. infections.
In the present study, ST1, ST3, and ST4 were detected in brown rats. ST4 was the most prevalent, and it was also found in cats and dogs. Generally, rodents have been shown to be the chief animal reservoir of ST4, which is an ancestry with recent entry into the human population $[63,64]$. ST4 has been the most prevalent subtype found in Danish Blastocystis-positive patients presenting with acute diarrhoea [55]. Interestingly, ST4 is reported at low prevalence rates in asymptomatic, apparently healthy individuals in Spain [49]. Most epidemiological studies describing ST4 in human populations depict allele 42, allele 91, and allele 133 of this subtype [49, 65]. However, the ST4 genetic variant reported in rats here is allele 92. This is an interesting point that should be further investigated. In addition, ST4 has been found in NHPs, giraffes, kangaroos, dogs, pigs and ostriches [20, 33, 57, 61, 63]. Moreover, ST3 and ST4 were identified in cockroaches $[41,61,66]$.

In the present survey, ST7 and ST8 were also detected in dogs. ST7 is one of the most frequent subtypes in birds and is principally considered an avian subtype [57,62]. In addition to birds, ST7 has been reported in pigs, goats and dogs [22, 33, 56, 58]. Furthermore, ST8 has been reported more prevalently in NHP handlers, suggesting a zoonotic scatter from primates to their handlers [61, 63]. Recently, ST8 was identified in pigs and marsupials [20, 61].

ST10, detected in the present study both in dogs and cats, has been reported exclusively in non-human hosts, including wild animals and livestock (cattle, yaks, camels, pigs, sheep and goats) in Libya, UK, China, Malaysia, Japan and the UAE $[15,56,58,59,67-70]$. ST10 was also detected in cats from the USA and dogs from France [7, 71].

Finally, we detected ST14 in two cats. ST14 is similar to ST10 in terms of host range, infecting some common livestock and some artiodactyls [57, 68]. Recently, ST14 was isolated from hooded crows and pigeons in Iran [29].

In dogs, ST1 to ST3 have been identified in Italy [50], France [71], Australia [72], and the Philippines [73], suggesting that dogs could be involved in the transmission of Blastocystis sp. to humans. Dogs are proficient in shedding possibly zoonotic subtypes and may act as incidental zoonotic reservoirs for infection. Particularly, direct evidence of zoonotic transmission has been granted by other surveys examining human and canine/feline 
$81-$ MN264511 Blastocystis sp. (ST1-Rat-This Study)

92 MN264518 Blastocystis sp. (ST1-Cat-This Study)

62 AY135404 Blastocystis sp. (ST1-Pig-France)

97 AB091240 Blastocystis sp. (ST1-Chicken-Japan)

$47 \$ 4$ AB023499 Blastocystis sp. (ST1-Human-Japan)

476 AB107961 Blastocystis sp. (ST1-Pig-Japan)

93 EU445490 Blastocystis sp. (ST1-Monkey-Philippines)

[ EU445486 Blastocystis sp. (ST1-Pig-Philippines)

$68 \$ 5$ AB107967 Blastocystis sp. (ST1-Orangutan-Japan)

82 MN264512 Blastocystis sp. (ST2-Dog-This Study)

52 EU445487 Blastocystis sp. (ST2-Pig-Philippines)

- MK801368 Blastocystis sp. (ST2-Pig-Germany)

99 - EU445491 Blastocystis sp. (ST2-Monkey-Philippines)

49 [AB107969 Blastocystis sp. (ST2-Macaque-Japan)

56 EU427518 Blastocystis sp. (ST2-Wallaby-Japan)

GU256932 Blastocystis sp. (ST11-Elephant-Australia)

- GU256907 Blastocystis sp. (ST11-Elephant-Belgium)

$2982 \quad 100$ GU256929 Blastocystis sp. (ST11-Elephant-Netherlands) 99 AB091246 Blastocystis sp. (ST7-Quail-Japan)

99 AY135412 Blastocystis sp. (ST7-Duck-France)

$99-$ AB107973 Blastocystis sp. (ST7-Swan Goose-Japan)

${ }_{100}$ AY135410 Blastocystis sp. (ST7-Chicken-France)

$97 \quad 97-\mathrm{AF} 408426$ Blastocystis sp. (ST9-Human-Japan)

91 KC138681 Blastocystis sp. (ST9-Human-Denmark) 37 AB091243 Blastocystis sp. (ST6-Quail-Japan)

100 AB107972 Blastocystis sp. (ST6-Partridge-Japan)

99 - AB070994 Blastocystis sp. (ST6-Chicken-Japan)

95 EU445485 Blastocystis sp. (ST6-Chicken-Philippines)

95 - AY135411 Blastocystis sp. (ST6-Turkey-France)

100 KC148209 Blastocystis sp. (ST13-Mousedeer-England)

GU256934 Blastocystis sp. (ST13-Quokka-Australia)

00, AB070998 Blastocystis sp. (ST5-Pig-Japan)

97 AB107966 Blastocystis sp. (ST5-Cattle-Japan)

99 MN264522 Blastocystis sp. (ST14-Cat-This Study)

$40 \quad 559$ KC148205 Blastocystis sp. (ST14-Cattle-England)

KC148206 Blastocystis sp. (ST14-Mouflon-England)

54 EU427515 Blastocystis sp. (ST12-Wallaby-Japan)

GU256906 Blastocystis sp. (ST12- Giraffe-Australia)

$83-$ GU256924 Blastocystis sp. (ST12- Giraffe-Netherlands)

63 MN264515 Blastocystis sp. (ST8-Dog-This Study)

80 KX234612 Blastocystis sp. (ST8-Dog-Malaysia)

KX234613 Blastocystis sp. (ST8-Dog-Malaysia)

108 [ KX234601 Blastocystis sp. (ST8-Goat-Malaysia)

AB107971 Blastocystis sp. (ST8-Pheasant-Japan)

83

AB107970 Blastocystis sp. (ST8-Ruffed Lemur-Japan)

66 JQ974945 Blastocystis sp. (ST8-Chimpanzee-Czech Republic)

89 MN264517 Blastocystis sp. (ST10-Dog-This Study)

MN264521 Blastocystis sp. (ST10-Cat-This Study)

KC148207 Blastocystis sp. (ST10-Camel-Libya)

100 KX352006 Blastocystis sp. (ST10-Fish-Malaysia)

KX234638 Blastocystis sp. (ST10-Goat-Malaysia)

82 KX234639 Blastocystis sp. (ST10-Bird-Malaysia)

KX234616 Blastocystis sp. (ST10-Dog-Malaysia)

74

EU427516 Blastocystis sp. (ST4-Tree Kanga

Blastocystis sp. (ST4-Human-USA)

KX234634 Blastocystis sp. (ST4-Dog-Malaysia)

KX234618 Blastocystis sp. (ST4-Dog-Malaysia)

26 MN264514 Blastocystis sp. (ST4-Dog-This Study)

46 - MN264509 Blastocystis sp. (ST4-Rat-This Study)

49 MN264520 Blastocystis sp. (ST4-Cat-This Study)

MN264510 Blastocystis sp. (ST3-Rat-This Study)

91 LN264519 Blastocystis sp. (ST3-Cat-This Study)

AB107965 Blastocystis sp. (ST3-Cattle-Japan)

MK801366 Blastocystis sp. (ST3-Pig-Germany)

500 MK801366 Blastocystis sp. (ST3-Pig-Germany)

98

- EU445489 Blastocystis sp. (ST3-Monkey-Philippines)

39 - MN264513 Blastocystis sp. (ST3-Dog-This Study)

${ }_{70}$ KX234611 Blastocystis sp. (ST3-Dog-Malaysia)

EU427512 Blastocystis sp. (ST16- Red Kangaroo-Japan)

100 EU427514 Blastocystis sp. (ST16- Red Kangaroo-Japan)

KC148208 Blastocystis sp. (ST17-Gundi-Libya)

KC148211 Blastocystis sp. (ST15-Gibbon-England)

100 KC148210 Blastocystis sp. (ST15-Camel-Libya)

U37108 Proteromonas lacertae 
populations living in the same spatiotemporal setting. In a prior study, eight dogs in close contact with 11 symptomatic family members infected by Blastocystis sp. were all positive for the parasite and harboured at least one subtype shared with each of the corresponding patients, suggesting that the source of infection of the owners was their household dogs [72]. Blastocystis ST2 to ST5 were concurrently found in people and domestic dogs living in an urban community in the Philippines [73]. Likewise, ST5 was identified in a villager and a dog both living in a village in Thailand signifying the probability of dogs as sources of zoonotic transmission of Blastocystis sp. [74]. Moreover, all Blastocystis sp. isolated from dogs in India and Indonesia were characterized as zoonotic subtypes $[33,75]$. Contrarily, a recent study demonstrate that pet dogs and cats play a tiny role as natural reservoirs of human Blastocystis sp. infection in Spain [49].

Specifically, stray dogs, which generally live in areas with poor sanitation and hygiene, were identified to be at greater risk of transferring Blastocystis sp. than domestic dogs [75]. Stray dogs in India carried a diverse range of Blastocystis sp. subtypes, including ST1 and ST4 to ST6, while Australian and Cambodian dogs harboured only ST1 and ST2, respectively [33]. Furthermore, ST1 was found in shelter-resident dogs and cats in the Pacific Northwest of the USA, emphasizing the potential role of stray dogs and cats as the origin of human Blastocystis sp. infection [7].

People who have close contact with dogs and cats for occupational or leisure reasons are at a high risk of acquiring Blastocystis sp. infection. Faced with the fact that coprophagia is a common instinct in dogs, the greater prevalence and diversity of subtypes found in dogs could be ascribed to their increased environmental exposure to faecal content from humans, cattle, sheep, goats and birds. Meanwhile, Blastocystis sp. in animal faeces can enter creeks and rivers by surface run-off after extreme rainfall, which allows water contamination downstream and broad geographical dispersion of Blastocystis sp. The environmental pollution level, size of cysts, resistance of cysts to water treatment methods such as chlorination, and poor elimination of cysts during the filtration process permit the zoonotic transmission of Blastocystis sp. [76].

\section{Conclusions}

The findings of this study showed that dogs, cats, and rats in Fars Province, Iran are shedding Blastocystis sp. Eight subtypes were characterized, with subtype overlaps between animals. Dogs, cats and rats are presumed to be part of the transmission dynamics of this infection in Fars Province. Although the role of these animals as a possible natural reservoir of Blastocystis sp. remains obscure, it seems that these animals could represent possible reservoirs of zoonotic transmission of Blastocystis sp. These data also accentuate the significance of screening other hosts of Blastocystis sp. in order to fully characterize the epidemiology of this protist. Given that extensive genetic diversity exists within Blastocystis sp. subtypes, future molecular characterization and comparisons of dog, cat, rat, human and other mammalian Blastocystis sp. subtypes using multilocus sequence typing (MLST) performed within communities where Blastocystis sp. is endemic in dogs, cats and humans will ideally shed further light on their role as natural hosts for infection. This epidemiological cohort study also furnishes essential information for taking preventive and control measures that should help lessen the risks of zoonotic transmission of Blastocystis sp. to humans.

\section{Supplementary information}

Supplementary information accompanies this paper at https://doi. org/10.1186/s13071-020-04225-9.

Additional file 1: Figure S1. The central-body form of Blastocystis sp. stained with Wheatley's trichrome stain (1000x magnification).

Additional file 2: Figure S2. Electrophoresis of PCR products of Blastocystis sp. DNA extracted from dog, cat, and rat faecal samples based on the 185 rRNA gene. The nine lanes contain the products from the negative control (Lane 9), positive control (Lane 8), positive samples of Blastocystis sp. that shows a specific diagnostic band of the 1100-bp fragment (Lanes 1-7), and a molecular size marker (Lane MM).

\section{Abbreviations}

ST: subtype; NHPS: non-human primates; TNR: trap, neuter, and release; SAF: sodium acetate-acetic acid-formalin; LYSGM: liver extract-yeast extract-serumgastric mucin; BSA: bovine serum albumin; NJ: neighbour-joining.

\section{Acknowledgements}

The authors would like to thank and appreciate all the veterinary staff throughout Fars Province, Iran, who contributed to the collection of animal faecal samples.

\section{Authors' contributions}

IM, ALG, MTM and MHM conceived and designed the study. IM, FBG and NM carried out the veterinary examination and laboratory work. IM and FBG analysed and interpreted the data. IM drafted the first version of the manuscript. IM, ALG and MTM critically reviewed the manuscript. All the authors read and approved the final manuscript.

Funding

No funding or sponsorship was received either for the study or article publication.

\section{Availability of data and materials}

All data generated or analysed during this study are included in this published article. Sequences were submitted to the GenBank database under the accession numbers MN264509-MN264522.

\section{Ethics approval and consent to participate}

The research protocol was reviewed and approved by the Institutional Animal Care Committee (IACC) of Shiraz University of Medical Sciences (No. 93-2817) for animal ethical standards. 


\section{Consent for publication \\ Not applicable.}

\section{Competing interests}

The authors declare that they have no competing interests.

\begin{abstract}
Author details
${ }^{1}$ Department of Medical Parasitology and Mycology, School of Medicine, Shiraz University of Medical Sciences, Shiraz, Iran. ${ }^{2}$ Department of Molecular Medicine, School of Advanced Medical Sciences and Technologies, Shiraz University of Medical Sciences, Shiraz, Iran. ${ }^{3}$ Department of Veterinary Medicine, Università degli Studi di Milano, Milan, Italy. ${ }^{4}$ Department of Medical Laboratory Sciences, Zeinab Hospital, Shiraz University of Medical Sciences, Shiraz, Iran.
\end{abstract}

Received: 27 April 2020 Accepted: 14 July 2020

Published online: 22 July 2020

\section{References}

1. Tan KSW. New insights on classification, identification, and clinical relevance of Blastocystis sp. Clin Microbiol Rev. 2008;21:639-65.

2. Zhang X, Zhang S, Qiao J, Wu X, Zhao L, Liu Y, Fan X. Ultrastructural insights into morphology and reproductive mode of Blastocystis hominis. Parasitol Res. 2012;110:1165-72.

3. El Safadi D, Gaayeb L, Meloni D, Cian A, Poirier P, Wawrzyniak I, et al. Children of Senegal River Basin show the highest prevalence of Blastocystis sp. ever observed worldwide. BMC Infect Dis. 2014;14:164.

4. Lee LI, Chye TT, Karmacharya BM, Govind SK. Blastocystis sp.: waterborne zoonotic organism, a possibility? Parasit Vectors. 2012:5:130.

5. Parkar U, Traub RJ, Vitali S, Elliot A, Levecke B, Robertson I, et al. Molecular characterization of Blastocystis isolates from zoo animals and their animalkeepers. Vet Parasitol. 2010;169:8-17.

6. Wang W, Owen H, Traub RJ, Cuttell L, Inpankaew T, Bielefeldt-Ohmann H. Molecular epidemiology of Blastocystis in pigs and their in-contact humans in Southeast Queensland, Australia, and Cambodia. Vet Parasitol. 2014;203:264-9.

7. Ruaux CG, Stang BV. Prevalence of Blastocystis in shelter-resident and client-owned companion animals in the US Pacific Northwest. PLoS ONE. 2014;9:e107496.

8. Casero RD, Mongi F, Sánchez A, Ramírez JD. Blastocystis and urticaria: examination of subtypes and morphotypes in an unusual clinical manifestation. Acta Trop. 2015;148:156-61.

9. Cifre S, Gozalbo M, Ortiz V, Soriano JM, Merino JF, Trelis M. Blastocystis subtypes and their association with irritable bowel syndrome. Med Hypotheses. 2018;116:4-9.

10. Yason JA, Liang YR, Png CW, Zhang Y, Tan KSW. Interactions between a pathogenic Blastocystis subtype and gut microbiota: in vitro and in vivo studies. Microbiome. 2019;7:30.

11. Lepczyńska M, Białkowska J, Dzika E, Piskorz-Ogórek K, Korycińska J. Blastocystis: how do specific diets and human gut microbiota affect its development and pathogenicity? Eur J Clin Microbiol Infect Dis. 2017;36:1531-40

12. Puthia MK, Lu J, Tan KSW. Blastocystis ratti contains cysteine proteases that mediate interleukin-8 response from human intestinal epithelial cells in an NF-kB-dependent manner. Eukaryot Cell. 2008:7:435-43.

13. Teo JDW, MacAry PA, Tan KSW. Pleiotropic effects of Blastocystis spp. subtypes 4 and 7 on ligand-specific toll-like receptor signaling and NF-KB activation in a human monocyte cell line. PLoS ONE. 2014;9:e89036.

14. Stensvold CR, Alfellani MA, Clark CG. Levels of genetic diversity vary dramatically between Blastocystis subtypes. Infect Genet Evol. 2012;12:263-73.

15. Alfellani MA, Taner-Mulla D, Jacob AS, Imeede CA, Yoshikawa H, Stensvold CR, Clark CG. Genetic diversity of Blastocystis in livestock and zoo animals. Protist. 2013;164:497-509.

16. Stensvold CR, Clark CG. Pre-empting Pandora's box: Blastocystis subtypes revisited. Trends Parasitol. 2020:36:229-32.

17. Alfellani MA, Stensvold CR, Vidal-Lapiedra A, Onuoha ES, Fagbenro-Beyioku AF, Clark CG. Variable geographic distribution of Blastocystis subtypes and its potential implications. Acta Trop. 2013;126:11-8.
18. Ramírez JD, Sánchez A, Hernández C, Flórez C, Bernal MC, Giraldo JC, et al. Geographic distribution of human Blastocystis subtypes in South America. Infect Genet Evol. 2016:41:32-5.

19. Yan Y, Su S, Ye J, Lai X, Lai R, Liao H, et al. Blastocystis sp. subtype 5: a possibly zoonotic genotype. Parasitol Res. 2007;101:1527-32.

20. Roberts T, Stark D, Harkness J, Ellis J. Subtype distribution of Blastocystis isolates from a variety of animals from New South Wales, Australia. Vet Parasitol. 2013;196:85-9.

21. Wang J, Gong B, Liu X, Zhao W, Bu T, Zhang W, et al. Distribution and genetic diversity of Blastocystis subtypes in various mammal and bird species in northeastern China. Parasit Vectors. 2018;11:522.

22. Wang J, Gong B, Yang F, Zhang W, Zheng Y, Liu A. Subtype distribution and genetic characterizations of Blastocystis in pigs, cattle, sheep and goats in northeastern China's Heilongjiang Province. Infect Genet Evol. 2018;57:171-6.

23. Calero-Bernal R, Santín M, Maloney JG, Martín-Pérez M, Habela MA Fernández-García JL, et al. Blastocystis sp. subtype diversity in wild carnivore species from Spain. J Eukaryot Microbiol. 2020;67:273-8.

24. Roberts T, Barratt J, Harkness J, Ellis J, Stark D. Comparison of microscopy, culture, and conventional polymerase chain reaction for detection of Blastocystis sp. in clinical stool samples. Am J Trop Med Hyg. 2011;84:308-12.

25. Ramírez JD, Sánchez LV, Bautista DC, Corredor AF, Flórez AC, Stensvold CR Blastocystis subtypes detected in humans and animals from Colombia. Infect Genet Evol. 2014:22:223-8.

26. Stensvold CR, Ahmed UN, Andersen LO, Nielsen HV. Development and evaluation of a genus-specific, probe-based, internal-process-controlled real-time PCR assay for sensitive and specific detection of Blastocystis spp. J Clin Microbiol. 2012;50:1847-51

27. Betts EL, Gentekaki E, Thomasz A, Breakell V, Carpenter Al, Tsaousis AD. Genetic diversity of Blastocystis in non-primate animals. Parasitology. 2018;145:1228-34.

28. Badparva E, Ezatpour B, Mahmoudvand H, Behzadifar M, Behzadifar M, Kheirandish F. Prevalence and genotype analysis of Blastocystis hominis in Iran: a systematic review and meta-analysis. Arch Clin Infect Dis. 2017:12:e36648.

29. Asghari A, Sadraei J, Pirestani M, Mohammadpour I. First molecular identification and subtype distribution of Blastocystis sp. isolated from hooded crows (Corvus cornix) and pigeons (Columba livia) in Tehran Province. Iran. Comp Immunol Microbiol Infect Dis. 2019;62:25-30.

30. Badparva E, Sadraee J, Kheirandish F. Genetic diversity of Blastocystis isolated from cattle in Khorramabad, Iran. Jundishapur J Microbiol. 2015;8:e14810

31. Solaymani-Mohammadi S, Rezaian M, Hooshyar H, Mowlavi GR, Babaei Z, Anwar MA. Intestinal protozoa in wild boars (Sus scrofa) in western Iran. J Wildl Dis. 2004;40:801-3.

32. Clark CG, Stensvold CR. Blastocystis: isolation, xenic cultivation, and cryopreservation. Curr Protoc Microbiol. 2016;43:20A.1.1-8

33. Wang W, Cuttell L, Bielefeldt-Ohmann H, Inpankaew T, Owen H, Traub RJ. Diversity of Blastocystis subtypes in dogs in different geographical settings. Parasit Vectors. 2013:6:215.

34. Kumar S, Stecher G, Li M, Knyaz C, Tamura K. MEGA X: molecular evolutionary genetics analysis across computing platforms. Mol Biol Evol. 2018;35:1547-9.

35. Noël C, Dufernez F, Gerbod D, Edgcomb VP, Delgado-Viscogliosi P, Ho LC, et al. Molecular phylogenies of Blastocystis isolates from different hosts: implications for genetic diversity, identification of species, and zoonosis. J Clin Microbiol. 2005:43:348-55.

36. Stensvold CR, Nielsen HV, Mølbak K, Smith HV. Pursuing the clinical significance of Blastocystis-diagnostic limitations. Trends Parasitol. 2009;25:23-9

37. Stensvold CR, Lewis HC, Hammerum AM, Porsbo LJ, Nielsen SS, Olsen $\mathrm{KE}$, et al. Blastocystis: unravelling potential risk factors and clinical significance of a common but neglected parasite. Epidemiol Infect. 2009;137:1655-63.

38. Ajjampur SSR, Tan KSW. Pathogenic mechanisms in Blastocystis spp.-interpreting results from in vitro and in vivo studies. Parasitol Int. 2016;65:772-9.

39. Clark CG, van der Giezen M, Alfellani MA, Stensvold CR. Recent developments in Blastocystis research. Adv Parasitol. 2013;82:1-32. 
40. Alfellani MA, Jacob AS, Perea NO, Krecek RC, Taner-Mulla D, Verweij JJ, et al. Diversity and distribution of Blastocystis sp. subtypes in non-human primates. Parasitology. 2013;140:966-71.

41. Yoshikawa H, Koyama Y, Tsuchiya E, Takami K. Blastocystis phylogeny among various isolates from humans to insects. Parasitol Int. 2016;65:750-9.

42. Yoshikawa H, Abe N, Wu Z. PCR-based identification of zoonotic isolates of Blastocystis from mammals and birds. Microbiology. 2004;150:1147-51.

43. Vaisusuk K, Saijuntha W, Sedlak S, Thanchomnang T, Pilap W, Suksavate W, et al. Blastocystis subtypes detected in long-tailed macaques in Thailandfurther evidence of cryptic host specificity. Acta Trop. 2018;184:78-82.

44. Růžková J, Květoňová D, Jirků M, Lhotská Z, Stensvold CR, Parfrey LW, et al. Evaluating rodent experimental models for studies of Blastocystis ST1. Exp Parasitol. 2018;191:55-61.

45. Defaye M, Nourrisson C, Baudu E, Warwzyniak I, Bonnin V, Bonnet M, et al. Efficient and reproducible experimental infections of rats with Blastocystis spp. PLOS ONE. 2018;13:e0207669.

46. Yoshikawa H, Wu Z, Pandey K, Pandey BD, Sherchand JB, Yanagi T, Kanbara H. Molecular characterization of Blastocystis isolates from children and rhesus monkeys in Kathmandu, Nepal. Vet Parasitol. 2009;160:295-300.

47. Lee IL, Tan TC, Tan PC, Nanthiney DR, Biraj MK, Surendra KM, Suresh KG. Predominance of Blastocystis sp. subtype 4 in rural communities, Nepal. Parasitol Res. 2012;110:1553-62.

48. Abe N, Nagoshi M, Takami K, Sawano Y, Yoshikawa H. A survey of Blastocystis sp. in livestock, pets, and zoo animals in Japan. Vet Parasitol. 2002;106:203-12.

49. Paulos S, Köster PC, de Lucio A, Hernández-de-Mingo M, Cardona GA Fernández-Crespo JC, et al. Occurrence and subtype distribution of Blastocystis sp. in humans, dogs and cats sharing household in northern Spain and assessment of zoonotic transmission risk. Zoonoses Public Health. 2018:65:993-1002.

50. Gazzonis AL, Marangi M, Zanzani SA, Villa L, Giangaspero A, Manfredi MT. Molecular epidemiology of Blastocystis sp. in dogs housed in Italian rescue shelters. Parasitol Res. 2019;118:3011-7.

51. López J, Abarca K, Paredes P, Inzunza E. Intestinal parasites in dogs and cats with gastrointestinal symptoms in Santiago, Chile. Rev Med Chil. 2006;134:193-200.

52. Chai Y, Deng L, Liu H, Yao J, Zhong Z, Fu H, et al. First subtyping of Blastocystis sp. from pet rodents in southwestern China. Int J Parasitol Parasites Wildl. 2020;11:143-8.

53. Farah Haziqah MT, Mohd Zain SN, Chandrawathani P, Premaalatha B, Mohd Khairul Nizam MK, Arutchelvan R, Suresh K. Genetic diversity of rodent Blastocystis sp. from Peninsular Malaysia. Trop Biomed. 2018;35:586-92.

54. Jones MS, Whipps CM, Ganac RD, Hudson NR, Boorom K. Association of Blastocystis subtype 3 and 1 with patients from an Oregon community presenting with chronic gastrointestinal illness. Parasitol Res. 2009;104:341-5.

55. Stensvold CR, Christiansen DB, Olsen KEP, Nielsen HV. Blastocystis sp. subtype 4 is common in Danish Blastocystis-positive patients presenting with acute diarrhea. Am J Trop Med Hyg. 2011;84:883-5.

56. Song JK, Yin YL, Yuan YJ, Tang H, Ren GJ, Zhang HJ, et al. First genotyping of Blastocystis sp. in dairy, meat, and cashmere goats in northwestern China. Acta Trop. 2017;176:277-82.

57. Cian A, El Safadi D, Osman M, Moriniere R, Gantois N, Benamrouz-Vanneste $\mathrm{S}$, et al. Molecular epidemiology of Blastocystis sp. in various animal groups from two French zoos and evaluation of potential zoonotic risk. PLOS ONE. 2017;12:e0169659.

58. Song JK, Hu RS, Fan XC, Wang SS, Zhang HJ, Zhao GH. Molecular characterization of Blastocystis from pigs in Shaanxi province of China. Acta Trop. 2017;173:130-5.

59. Zhao GH, Hu XF, Liu TL, Hu RS, Yu ZQ, Yang WB, et al. Molecular characterization of Blastocystis sp. in captive wild animals in Qinling Mountains. Parasitol Res. 2017;116:2327-33.

60. Li WC, Wang K, Gu Y. Occurrence of Blastocystis sp. and Pentatrichomonas hominis in sheep and goats in China. Parasit Vectors. 2018;11:93.

61. Valença-Barbosa C, do Bomfim TCB, Teixeira BR, Gentile R, Neto SFdC, Magalhães BS, et al. Molecular epidemiology of Blastocystis isolated from animals in the state of Rio de Janeiro, Brazil. PLoS ONE. 2019;14:e0210740.
62. Greige S, El Safadi D, Bécu N, Gantois N, Pereira B, Chabé M, et al. Prevalence and subtype distribution of Blastocystis sp isolates from poultry in Lebanon and evidence of zoonotic potential. Parasit Vectors. 2018;11:389.

63. Stensvold CR, Alfellani MA, Nørskov-Lauritsen S, Prip K, Victory EL, Maddox C, et al. Subtype distribution of Blastocystis isolates from synanthropic and zoo animals and identification of a new subtype. Int J Parasitol. 2009;39:473-9.

64. Katsumata M, Yoshikawa H, Tokoro M, Mizuno T, Nagamoto T, Hendarto $J$, et al. Molecular phylogeny of Blastocystis isolates from wild rodents captured in Indonesia and Japan. Parasitol Res. 2018;117:2841-6.

65. Jiménez PA, Jaimes JE, Ramírez JD. A summary of Blastocystis subtypes in North and South America. Parasit Vectors. 2019:12:376.

66. Yoshikawa H, Wu Z, Howe J, Hashimoto T, Geok-Choo NG, Tan KSW. Ultrastructural and phylogenetic studies on Blastocystis isolates from cockroaches. J Eukaryot Microbiol. 2007;54:33-7.

67. Tan TC, Tan PC, Sharma R, Sugnaseelan S, Suresh KG. Genetic diversity of caprine Blastocystis from Peninsular Malaysia. Parasitol Res. 2013;112:85-9.

68. Masuda A, Sumiyoshi T, Ohtaki T, Matsumoto J. Prevalence and molecular subtyping of Blastocystis from dairy cattle in Kanagawa, Japan. Parasitol Int. 2018;67:702-5.

69. Ren M, Song JK, Yang F, Zou M, Wang PX, Wang D, et al. First genotyping of Blastocystis in yaks from Qinghai Province, northwestern China. Parasit Vectors. 2019;12:171.

70. AbuOdeh R, Ezzedine S, Madkour M, Stensvold CR, Samie A, Nasrallah $\mathrm{G}$, et al. Molecular subtyping of Blastocystis from diverse animals in the United Arab Emirates. Protist. 2019;170:125679.

71. Osman M, Bories J, El Safadi D, Poirel MT, Gantois N, Benamrouz-Vanneste $\mathrm{S}$, et al. Prevalence and genetic diversity of the intestinal parasites Blastocystis sp. and Cryptosporidium spp. in household dogs in France and evaluation of zoonotic transmission risk. Vet Parasitol. 2015;214:167-70.

72. Nagel R, Cuttell L, Stensvold CR, Mills PC, Bielefeldt-Ohmann H, Traub RJ. Blastocystis subtypes in symptomatic and asymptomatic family members and pets and response to therapy. Intern Med J. 2012;42:1187-95.

73. Belleza ML, Reyes JC, Tongol-Rivera PN, Rivera WL. Subtype analysis of Blastocystis sp. isolates from human and canine hosts in an urban community in the Philippines. Parasitol Int. 2016;65:291-4.

74. Parkar U, Traub RJ, Kumar S, Mungthin M, Vitali S, Leelayoova S, et al. Direct characterization of Blastocystis from feces by PCR and evidence of zoonotic potential. Parasitology. 2007;134:359-67.

75. Yoshikawa H, Tokoro M, Nagamoto T, Arayama S, Asih PBS, Rozi IE, Syafruddin D. Molecular survey of Blastocystis sp. from humans and associated animals in an Indonesian community with poor hygiene. Parasitol Int. 2016;65:780-4.

76. Suresh K, Smith HV, Tan TC. Viable Blastocystis cysts in Scottish and Malaysian sewage samples. Appl Environ Microbiol. 2005;71:5619-20.

\section{Publisher's Note}

Springer Nature remains neutral with regard to jurisdictional claims in published maps and institutional affiliations.

Ready to submit your research? Choose BMC and benefit from

- fast, convenient online submission

- thorough peer review by experienced researchers in your field

- rapid publication on acceptance

- support for research data, including large and complex data types

- gold Open Access which fosters wider collaboration and increased citations

- maximum visibility for your research: over 100M website views per year

At BMC, research is always in progress.

Learn more biomedcentral.com/submissions 